\title{
Phosphorus metabolites in the human placenta estimated in vivo by magnetic resonance spectroscopy
}

\author{
A $M$ Weindling, R D Griffiths, A S Garden, P A Martin, R H T Edwards
}

\begin{abstract}
Normal human placental metabolism has been studied in vivo by image localised ${ }^{31} \mathbf{P}$ magnetic resonance spectroscopy in 13 women with anterior placentas; five, however, were too fat for useful spectral signals to be obtained. Magnetic resonance spectra of good quality which were considered to have arisen from the placenta were obtained from seven women with uncomplicated pregnancies (median gestational age 35 weeks, range 28-39). One other woman had a twin pregnancy in which one fetus had died a few days before. The phosphodiester signal from the placenta of the dead fetus was outside the $95 \%$ confidence intervals for normal placentas, suggesting that this technique may potentially be useful in the assessment of placental function.
\end{abstract}

The ability to assess fetal wellbeing reliably is essential for making informed decisions about the management of the fetus that is considered to be at risk. The ideal method should be safe, non-invasive, and capable of providing information in normal as well as abnormal pregnancies. Indirect methods, such as Doppler blood flow studies, are neither specific nor sensitive enough to diagnose a hypoxic fetus accurately. Direct methods, such as cordocentesis, are invasive and risky.

Magnetic resonance (MR) spectroscopy, although expensive, has the potential for providing information about fetal wellbeing. ${ }^{1}$ Earlier in vitro work suggested that MR could have a role in the study of placental disorders. ${ }^{2}$

Following a brief preliminary report, ${ }^{3}$ we present the first observations of human placental metabolism assessed non-invasively by ${ }^{31} \mathrm{P} M R$ spectroscopy.

PO Box 47,

Liverpool L69 3BX,

Department of

Child Health

A $M$ Weindling

Department of Medicine

R D Griffiths

R H T Edwards

Department of Obstetrics and Gynaecology

A S Garden

Magnetic Resonance

Research Centre

P A Martin

Correspondence to:

Dr Weindling.

Accepted 7 January 1991 made to image fetuses; none has had to be stopped because of physical discomfort and only one because of claustrophobia.
LOCALISATION OF THE FETUS AND PLACENTA The fetus was imaged using a fast scan technique that has previously been described. ${ }^{4}$ The precise position of the placenta in relation to the anterior abdominal wall, the uterus, and the fetus was established in at least two orthogonal views using the following settings: repetition time $25 \mathrm{msec}$, echo delay time $12 \mathrm{msec}$, and a flip angle of $10^{\circ}$.

\section{MAGNETIC RESONANCE SPECTROSCOPY}

An $11 \mathrm{~cm}$ diameter transmit and receive surface coil was used to generate a ${ }^{31} \mathrm{P}$ spectrum from the placenta. The exact position of the surface coil on the anterior abdominal wall was confirmed by a small glycerol phantom built into the coil, which could be seen on the fast scan MR images.

The size and position of the coil were such that, from the images obtained, placental tissue could be seen extending at least as far as one coil's radius from the outer edge of the coil. The image based spectroscopy protocol, one dimensional chemical shift imaging (1D-CSI), was used to define contiguous slices $10 \mathrm{~mm}$ thick and parallel to the surface coil in the orthogonal plane. $^{5}$

The sensitive volume of our surface coil was checked by conventional imaging of a phantom, which was constructed to subject the coil to identical operating conditions (for example, coil loading) as those encountered for a pregnant woman. The radio frequency pulse power was chosen so that the sensitive volume produced was shaped like a shallow dome. At $4 \mathrm{~cm}$ depth-the depth at which the placenta was encountered-the volume was roughly a flat disk $7 \mathrm{~cm}$ in diameter. Together with the image based spectroscopy protocol (1D-CSI, repetition time 1 second, and 16 excitations with 128 phase encoding steps), this technique, which produced $1 \mathrm{~cm}$ thick slices parallel to the plane of the coil, gave a sensitive volume of about 38 $\mathrm{ml}$.

The number of phase encoding steps was unusual and was used for two reasons. Firstly, our computer software worked more efficiently on multiples of 128. Secondly, the number of phase encoding steps determined the number of data points in the spatial direction; the more such points there were, the better the spatial resolution.

Previous reproducibility studies at this centre using an identical technique to study the spleen 
found a coefficient of variation of metabolite ratios of between 2 and $14 \% .^{6}$

A study was considered satisfactory if at least two contiguous $1 \mathrm{~cm}$ slices could be localised within the placenta on both the axial and sagittal images. The MR signal that was analysed was obtained at $4 \mathrm{~cm}$ depth from the coil. Proton 1D-CSI spectra were collected to confirm localisation and for additional chemical shift reference. Spectra were processed with a Sun work station running the General Electric SA/GE spectroscopy software. Automated curve fitting was done with the Marquardt-Levenburg nonlinear least squares algorithm.

Each metabolite was expressed as a fraction of the total phosphorus signal. No attempt was made to correct for partial saturation effects which were invariably present because of the short repetition time used.

\section{PATIENTS}

Seven normal pregnant women (median gestational age 35 weeks, range 28-39) were studied. One other woman with a twin pregnancy in which one of the fetuses had died a few days before was also studied. These studies were approved by the ethics committee of this health authority, and were carried out within the guidelines of the National Radiological Protection Board of the United Kingdom.?

\section{Results}

It was possible to differentiate the spectra that originated from the anterior abdominal wall, the placenta and uterine wall, and the placenta alone at $4 \mathrm{~cm}$ depth (fig 1 ). The spectrum from
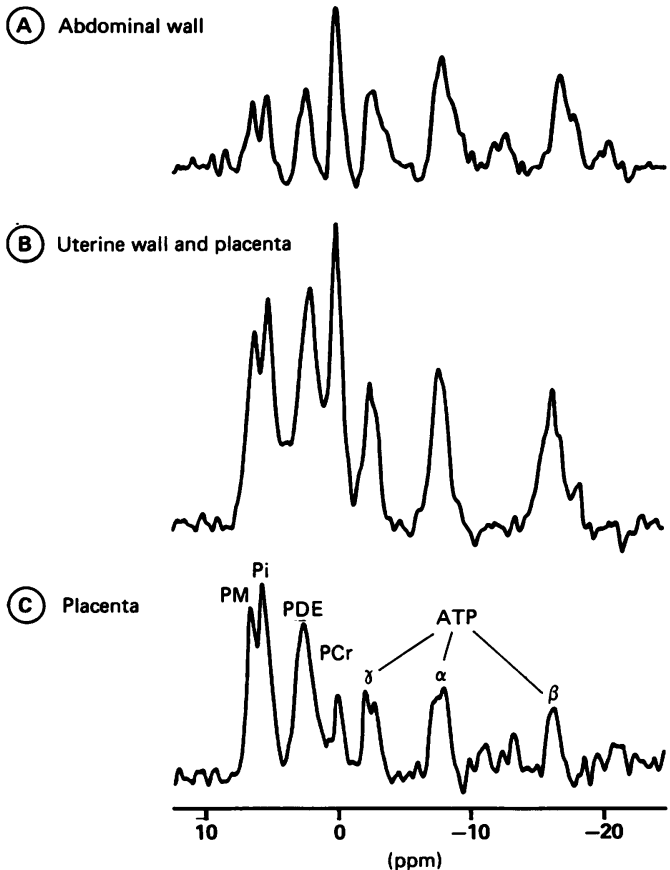

Figure 1 Image localised ${ }^{31} P$ spectrum obtained with one dimensional chemical shift imaging from $(A)$ the abdominal wall, $(B)$ uterine wall and placenta, and $(C)$ human placenta in vivo at 35 weeks' gestation. The placental spectrum was obtained from a slice $4 \mathrm{~cm}$ deep to the surface coil. PM, phosphomonoesters; $P i$, inorganic phosphate; $P C r$, phosphocreatine; and PDE, phosphodiesters.

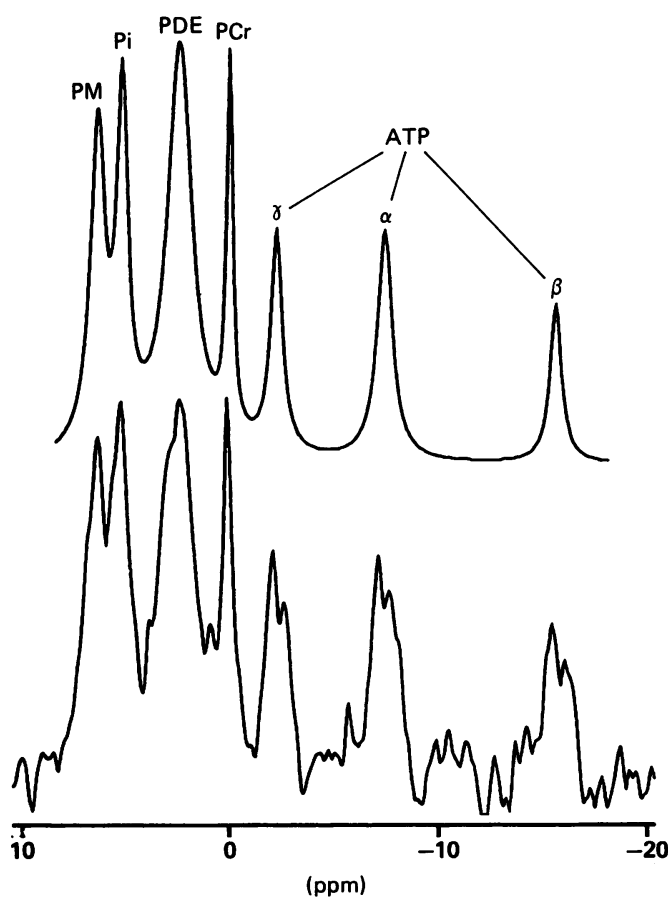

Figure $2{ }^{31} P$ spectrum from the placenta of the dead fetus. The raw signal is shown in the lower panel, with the smoothed signal generated by the computer assisted curve fitting program in the upper panel. PM, phosphomonoesters; $P i$, inorganic phosphate; $P C r$, phosphocreatine; and PDE, phosphodiesters.

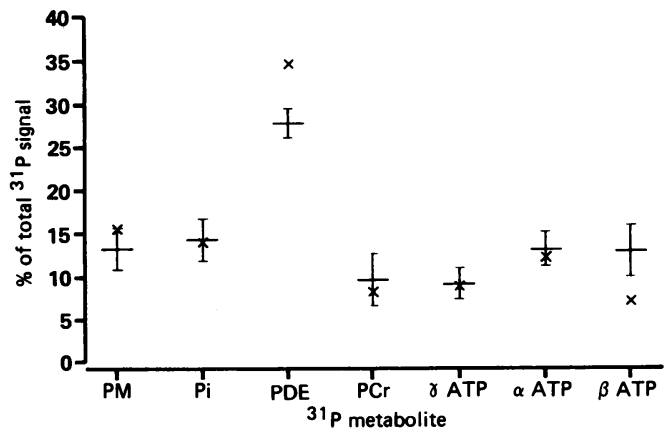

Figure 3 Quantitative ${ }^{31} P$ metabolite data from seven uncomplicated pregnancies expressed as a proportion of the total phosphorus signal; bars indicate mean $(95 \%$ confidence intervals). The results obtained from the placenta of the dead fetus are indicated separately $(X) . P M$, phosphomonoesters; $P i$, inorganic phosphate; $P C r$, phosphocreatine; and PDE, phosphodiesters.

the placenta of the dead fetus is shown in fig 2 .

Studies of five other women were abandoned because they were fatter, and as a result their placentas were deeper than the $4-5 \mathrm{~cm}$ required for optimal collection of signals.

The data from each of the seven normal subjects were combined to give a mean value with 95\% confidence intervals (CI). The phosphodiester signals from the placenta of the dead fetus fell outside the $95 \%$ CI (fig 3); although the $\beta$ ATP signal from the dead fetus was also low, the signal:noise ratio from this relatively small signal was poor, and we are therefore reluctant to place too much significance on this observation.

\section{Discussion}

These studies have shown that it is possible to obtain magnetic resonance spectra from anter- 
iorly placed placentas. There was no evidence of any systematic change in signal with gestation, and one reason for this is that the placenta is functionally mature by 28 weeks.

When placental function was grossly deranged, however (in the case of the intrauterine death), possible breakdown products were visible. Phosphomonoesters and phosphodiesters are representative of phospholipid metabolism. Their major constituents are the precursors of membrane constituents-that is, phosphorylethanolamine (a precursor of phosphatidylethanolamine) and phosphorylcholine (a precursor of phosphatidylcholine). Phosphomonoester signals might be expected to increase during early pregnancy and cell growth, and to decline when the placenta matures or earlier if growth ceases. The sugar phosphates, notably 2, 3-diphosphoglycerate in red blood cells, also contribute to the phosphomonoester signal. The phosphodiester resonance arises from contributions from glycerophosphorylethanolamine and glycerophosphorylcholine, which are the degradation products of phospholipids and myelin.

As predicted by Dawson $e t a l,{ }^{8}$ the ability to detect in vivo large resonances from inorganic phosphorus and the products of phospholipid synthesis and catabolism seems to offer the opportunity to study placental function and its disorders. The relatively larger amounts of phosphodiesters that were observed in the case of the placenta of the dead fetus were much what might have been expected in theory.

We are aware that our method of localising to a slice allowed some contamination of the signal, both from neighbouring tissues and from non-placental tissue at the ends of the slice. This was in part restricted by the sensitivity of the surface coil, but there was inevitably some contamination (probably less than 10\%) from myometrium or abdominal wall and this may have accounted for the fairly large phosphocreatine signal. The enzyme creatine kinase has been described in placental tissue and small amounts of phosphocreatine are present on placental extract analysis. ${ }^{9}$ The absence of phosphocreatine from in vitro studies may have been the result of hydrolysis. The much larger phosphodiester signal is more interesting, and cannot be accounted for by myometrial contamination; the finding is in contrast with in vitro studies that showed a low content. ${ }^{10}$ One explanation has come from studies of hepatic phosphodiester metabolism by Bates et al. ${ }^{11}$ They suggested that a signal caused by the phospholipid component of the membranes is seen at the lower field strength at which in vivo studies are carried out; this is lost at the higher fields used in the in vitro analyses because of chemical shift anisotropy in an underlying broad hump. ${ }^{11}$

Caution must therefore be exercised in predicting possible in vivo changes from in vitro studies for two reasons. Firstly, because of the different field strengths. Secondly, because of the rapid reduction in ATP concentration in the human placenta after delivery, which was shown by Carroll and Young, who suggested that biochemical deterioration may occur within 30 seconds of delivery. ${ }^{12}$

The safety of any technique used in pregnancy is of paramount importance. Safety aspects of this technique were considered carefully. National Radiology Protection Board guidelines relate to exposure to radio frequency power. A conventional magnetic resonance image is one tenth of that specified in the guidelines. ${ }^{7}$ The fast scan imaging technique used in these studies required one hundredth of the energy of conventional images. Magnetic resonance spectroscopy required one tenth of the energy of fast scan images, that is one tenthousandth of that specificed in the guidelines. Cell cultures and animal studies have also been done to assess the safety of MR, and studies of pregnant mice showed no differences in litter numbers or growth rates in animals exposed to MR compared with controls. ${ }^{12}$ Follow up studies of patients and volunteers who have undergone imaging also failed to show any hazards. ${ }^{13}$

This technique is still some way from being clinically useful. Improved coil design to provide a more homogeneous transmitting field will improve localisation and allow comparison of slices recorded at different depths.

We gratefully acknowledge support from Action Research for the Crippled Child.

1 Griffiths RD, Edwards RHT. The biomedical applications of spectroscopy and spectrally resolved imaging. In: Wehrli F, Kneeland WP, Shaw D, eds. Principles and applications of biomedical magnetic resonance imaging. USA: $\mathrm{VCH}$ Publishers, 1988:521-51

2 Noyszewski EA, Raman J, Trupin SR, McFarlin BL, Dawson MJ. Phosphorus 31 nuclear magnetic resonance examination of female reproductive tissues. Am $\mathcal{J}$ Obste Gynecol 1989;161:282-8.

3 Griffiths RD, Weindling AM, Garden AS, Martin PA, Edwards RHT. Image guided ${ }^{31} \mathrm{P}$ MR spectroscopy of the human placenta. Society of Magnetic Resonance Medicine Abstracts 1989;2:582.

4 Garden AS, Griffiths RD, Weindling AM, Martin PA. Fast scan magnetic resonance imaging in fetal visualisation. $A m$ $\mathcal{f}$ Obstet Gynecol (in press).

5 Glayzer GM, Smith SR, Chevenert TL, Martin PA, Stevens AN, Edwards RHT. Image localized ${ }^{31} \mathrm{P}$ magnetic resonAN, Edwards RHT. Image localized P magnetic resonance spectroscopy of the human liver.
Resonance in Biomedicine 1989;1:184-9.

6 Smith SR, Martin PA, Davies JM, Edwards RHT. Charac terisation of the spleen by in vivo image guided 31P magnetic resonance spectroscopy. Nuclear Magnetic Resonance in Biomedicine 1989;2:172-8.

National Radiological Protection Board. Revised guide-lines on acceptable limits of exposure during NMR clinical imaging. $B r \mathcal{F}$ Radiol 1981;138:159-60.

8 Dawson MJ, McFarlane DK, McFarlin BL, Noyszewski EA, Trupin SR. The biochemistry of female reproductive tissues studied by 31 phosphorus nuclear magnetic resonance spectroscopy: effects of pregnancy, hormonal manipulation, and disease. Biol Reprod 1988;38:31-8.

Kay $\mathrm{HH}$, Gordon JD, Ribeiro AA, Spicer LD. $31 \mathrm{P}$ NMR spectroscopy studies of human placenta and placental sectroscopy studies or human placenta and placental 1988;1:475.

10 Robbie D, Foster MA. Pulsed magnetic field exposure during pregnancy and implications for NMR foetal imaging: pregnancy and implications for NMR foetal imaging: a study

11 Bates TE, Williams SR, Gadian DG. Hepatic phosphodiester signals in vivo: the importance of field strength. Society of signals in vivo: the importance of field strength. Socies

12 Carroll MJ, Young M. Observations on the energy and redox state and protein synthetic rate in animal and human state and protein synthetic rate in anim

13 Reid A, Smith FW, Hutchison JMS. Nuclear magnetic resonance imaging and its safety implications: follow up of 181 patients. $B r \mathcal{F}$ Radiol 1982;55:784-6. 